\title{
Evaluation of fetal arrhythmias by echocardiography
}

\author{
L D ALLAN, R H ANDERSON, I D SULLIVAN, S CAMPBELL, D W HOLT, M TYNAN \\ From Guy's Hospital; King's College Hospital, and the Cardiothoracic Institute, Fulham Road, London
}

SUMMARY The normal fetal heart shows variation in rate and rhythm. Twenty three cases that fell outside the normal patterns of variation of heart rate and rhythm were evaluated by echocardiography. Ten showed atrial or ventricular extrasystoles and these were not associated with perinatal morbidity or mortality. Seven cases showed complete heart block, two in association with structural cardiac abnormality. Six cases were of supraventricular tachycardia, five of which were successfully treated and one was delivered prematurely. It was possible to diagnose accurately the type of arrhythmia using the $\mathrm{M}$-mode echocardiogram to compare the relation between atrial and ventricular contraction. Cross-sectional echocardiography allowed identification of associated structural abnormalities. Recognition that an arrhythmia is present and appropriate prenatal treatment if this is indicated will avoid unnecessary operative or premature deliveries.

Variations in fetal heart rate and rhythm are noticed with greater frequency as the use of cardiotachography and ultrasonography increases in routine obstetric practice. The development of a specialised interest in fetal echocardiography has led to the referral for assessment of cases demonstrating such variations. We report the findings in 23 cases of fetal arrhythmia and describe the method of their evaluation.

The prognosis in each case of an arrhythmia is governed by the type of arrhythmia, the association with structural cardiac anomaly, or the coexistence of intrauterine cardiac failure. ${ }^{1}$ The identification of normal cardiac anatomy and of structural malformations in prenatal life have been described by several authors. ${ }^{2-4}$ Intrauterine cardiac failure, or nonimmune hydrops fetalis, has been observed in association with fetal arrhythmias. ${ }^{56}$ The degree of cardiac failure has been shown to respond to control of a tachycardia in prenatal life. ${ }^{7}$ We have found it possible to assess the type of arrhythmia in intrauterine life by the use of M-mode echocardiography. The type of arrhythmia is important not only in cases where therapeutic measures are indicated, but also in the management of the pregnancy and delivery.

\section{Subjects and methods}

An Advanced Technology Laboratories Mark III sector scanner was used for the cross-sectional and
M-mode echocardiographic study. The crosssectional study was performed initially in order to identify cardiac anatomy. The $M$ line was then directed in a standard fashion ${ }^{8}$ through the structures which were to be recorded. Atrial and ventricular wall motion were recorded, as were atrioventricular and arterial valve motion. Atrial systole was identified by the movement of the atrial wall towards the aortic root. This is seen in Fig. 1a. Ventricular systole was inferred from arterial valve opening or was identified by the onset of ventricular wall motion towards the septum. Ventricular contraction is shown in Fig. 1b. These mechanical events can establish the sequence of excitation by the temporal relation of atrial to ventricular contraction. The mechanical sequence of contraction was chosen to analyse the arrhythmias rather than the fetal electrocardiogram for several reasons. Firstly, the fetal electrocardiogram shows only QRS activity, the $P$ waves being of such small voltage that they are indiscernible. Secondly, the fetal electrocardiogram is not of sufficient quality to show changes in QRS morphology. Thirdly, the fetal electrocardiogram can only be reliably recorded from 18 to 28 weeks' gestation and from 34 weeks to term. In the youngest fetuses the fetal electrical signal is too weak to be recorded while in the 28 to 34 week fetuses the fetal electrical signal is said to be insulated from external recording by the vernix caseosa. ${ }^{9}$

Twenty-three patients were referred for assessment because routine antenatal observation, either clinical or by cardiotachography or ultrasonography, had detected an unusually fast, slow, or irregular fetal 


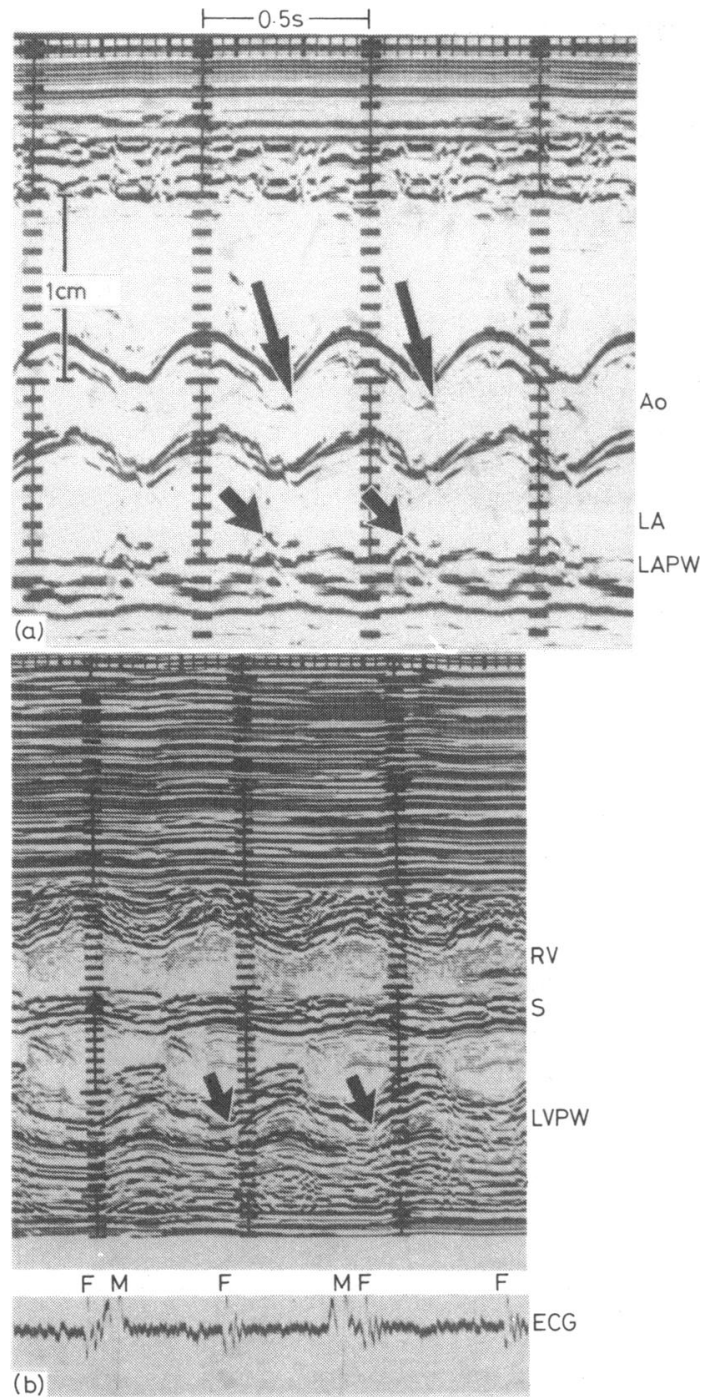

Fig. 1 (a) The M-mode echocardiogram is recorded through the aortic root and left atrial wall. Left ventricular systole can be inferred from the opening of the aortic valve (lange arrows). Atrial contraction occurs just before ventricular contraction and is indicated by the small arrows. (b) The M-mode echocardiogram is recorded across both ventricular chambers. The onset of ventricular systole occurs when either ventricular wall moves towards the interventricular septum, but the onset of left ventricular contraction is arrowed in the figure.

heart rhythm. In all cases the arrhythmia had been noted on more than one occasion.

\section{Results}

An arrhythmia was judged to be present when the heart rate or rhythm was outside the normal patterns of variation. These normal patterns have been reported in mid-trimester fetuses ${ }^{10}$ and in preterm infants. 11 They were also observed in our own echocardiographic study of 750 normal fetuses. The normal fetal heart rate is $140 \pm 20$ beats per minute at around 20 weeks' gestation, falling to $130 \pm 20$ beats per minute towards term. Normal variations in heart rate include episodes of bradycardia with heart rates of 70 to 100 beats per minute which last only three to four seconds. These episodes are often associated with fetal movement, are more common in the midtrimester, and decrease in frequency with advancing gestational age. In addition, short episodes of acceleration occur, with heart rates of between 160 and 180 beats per minute. These increase in frequency as pregnancy advances. Occasional atrial and ventricular extrasystoles causing irregular rhythms have also been described in up to $10 \%$ of fetuses and are thought to be part of normal variation. ${ }^{11} 12$ When extrasystoles were frequent, however, this was thought to merit assessment. Thus we considered that only a sustained bradycardia of less than 100 beats a minute, a sustained tachycardia of more than 200 beats a minute, or frequent, more than one in 10 , irregular beats constituted an arrhythmia to be analysed. The 23 cases presented here all fulfilled these criteria.

\section{IRREGULAR FETAL HEART RHYTHM}

Ten patients were referred in whom an irregular fetal heart rhythm had been shown by cardiotachography or real-time ultrasonography on more than one occasion. All the patients were in the last 10 weeks of pregnancy; in one case the irregularity was noticed during labour. One mother had uncontrolled maternal diabetes and the fetal rhythm disturbance decreased once adequate diabetic control was achieved. All the patients had frequent atrial and/or ventricular extrasystoles, at least one extrasystole occurring in every 10 beats. In one case the ventricular premature beat followed each sinus beat. Fig. 2 shows the echocardiographic findings in one case. There is an atrial and ventricular extrasystole recorded on this tracing. One premature ventricular contraction occurs after atrial contraction and is presumably an atrial premature contraction. The second premature ventricular contraction is unassociated with atrial wall motion and therefore is presumably a premature ventricular beat. Similar recordings of atrial and ventricular wall motion in the other cases studied showed the origin of premature beats to be predominantly atrial in six, ventricular in two, and mixed atrial and ventricular in one case. At no time did any case develop a tachycardia in association with premature beats. No case had associated intrauterine cardiac failure. All the arrhythmias disappeared 

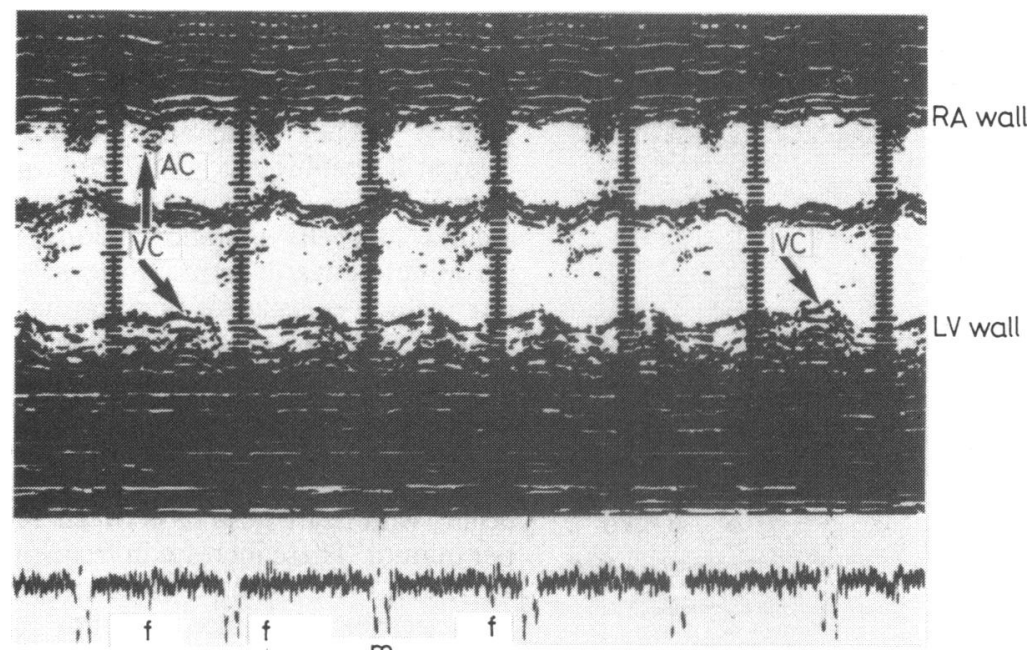

Fig. 2 The M-mode echocardiogram records atrial wall superiorly with ventricular wall motion inferiorly. The first premature ventricular contraction is seen to be associated with atrial contraction whereas the second premature ventricular contraction is unassociated with atrial wall motion. The first premature contraction is presumably therefore of atrial origin, whereas the second is presumably of ventricular origin and does not cause retrograde contraction of the atria.

before (eight cases) or just after delivery (two cases). All cases are alive and well after a minimum of six months follow up.

\section{COMPLETE HEART BLOCK}

Seven cases were referred because the fetal heart rate had been repeatedly recorded below 100 beats a minute. Two were associated with structural cardiac malformations. Both cases had left atrial isomerism and an atrioventricular septal defect. One case also had an interrupted aortic arch while the second had double outlet right ventricle and pulmonary stenosis. Despite the demonstration of the cardiac malformation and of complete heart block, a caesarian section was performed in the second case because of the slow fetal heart rate. In the other case the bradycardia had first been noticed before 12 weeks' gestational age.

The five remaining cases had anatomically normal hearts echocardiographically, but three mothers had clinical or serological evidence of connectiv's tissue disease. One case had severe clinical Sjögren's syndrome with serological confirmation; the other two cases had positive antinuclear and rheumatoid factors. Fig. 3 illustrates one of these cases and shows the atrial contraction to be approximately twice the ventricular rate, as evidenced by aortic valve opening. In all seven infants the heart rate was persistently in the range of 60 to 80 beats a minute, and the echocardiogram was diagnostic and similar to that shown in Fig. 3. Isolated heart block had a good prognosis.

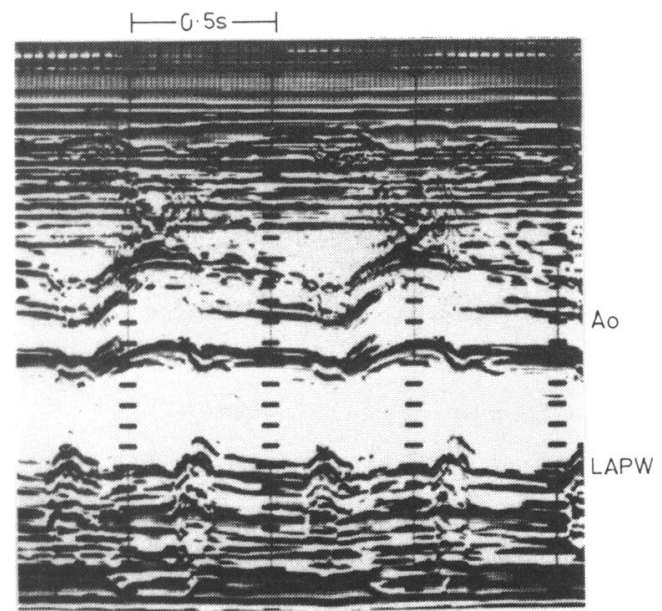

Fig. 3 The echocardiogram records the aortic root and left atrial wall. Atrial contraction is approximately twice as fast as ventricular contraction, as judged by aortic valve motion.

\section{FETAL TACHYCARDIAS}

There were three cases of atrial flutter and three of supraventricular tachycardia. There was no evidence of cardiac failure in five of the cases or of structural abnormality of the heart in any. The first case of atrial flutter presented at 33 weeks' gestation. The atrial rate was 400 a minute (Fig. 4). The ventricular rate was stable at 200 a minute. The lecithin sphing- 


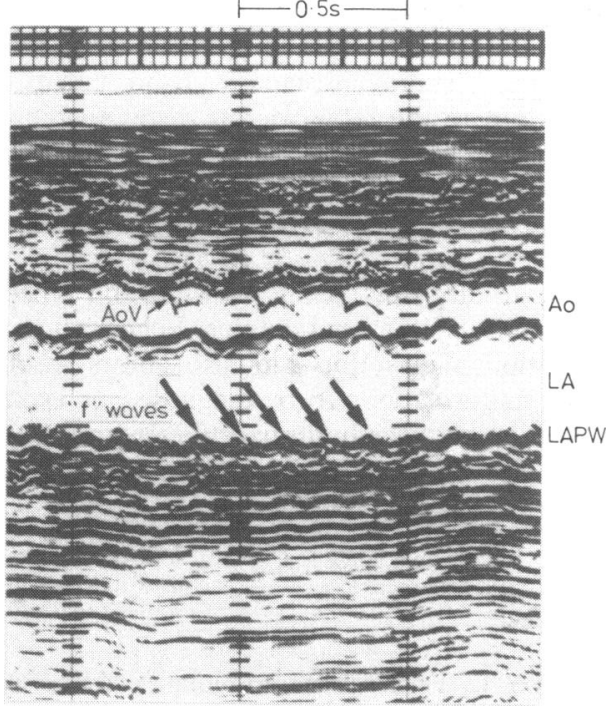

Fig. 4 The recording of left atrial wall motion shows flutter (' $\rho$ ') waves occurring at twice the ventricular rate which can be estimated from the aortic valve motion.

omyelin ratio suggested adequate fetal lung maturity for delivery, and labour was induced at 34 weeks' gestation. Cardioversion converted the atrial flutter to sinus rhythm soon after birth. Despite, however, apparently adequate lung maturity and steroid treatment to the mother before induction some complications of prematurity occurred. There was moderately severe respiratory distress syndrome and necrotising enterocolitis. The second case of atrial flutter presented at 34 weeks' gestation. The atrial rate was 440 a minute. During a half hour scan time the ventricular rate was either 220 or 440 beats a minute, the faster rate occurring during at least half of the scanning time. On the cross-sectional study the ventricles appeared to "quiver" while the fast rhythm occurred. The patient was digitalised, a dose of $0.25 \mathrm{mg}$ three times a day being necessary to achieve adequate maternal serum concentrations of digoxin $(1.6 \mu \mathrm{g} / \mathrm{l})$ and to achieve control of the fetal ventricular rate (below 200 beats a minute). One week before term the fetus was found to be in sinus rhythm. Delivery took place at term. Simultaneous maternal and cord blood estimations of serum concentrations of digoxin were 1.2 and $0.8 \mu \mathrm{g} / \mathrm{l}$, respectively. Atrial flutter has not recurred postnatally after six month follow-up. The third case of atrial flutter presented at 36 weeks' gestation. The ventricular rate was stable at 240 beats a minute but this rate, polyhydramnios, and fetal ascites had been observed for three weeks before referral. The fetus was severely hydropic. The rate was slowed to 200 a minute using digoxin $0.25 \mathrm{mg}$ three times a day

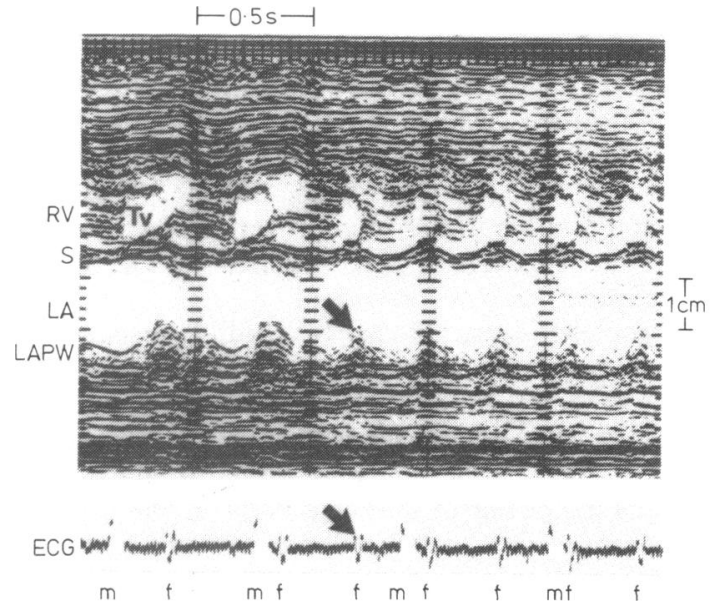

Fig. 5 The M-mode echocardiogram is recorded across the right ventricle and left atrial wall. The onset of supraventricular tachycardia is seen (arrowed) and can in this case be related to a fetal $Q R S$ complex on the fetal electrocardiogram recorded below. The premature atrial contraction occurs before ventricular contraction and starts a short burst of supraventricular tachycardia.

but this was causing toxic symptoms in the mother. Verapamil $10 \mathrm{mg}$ was given intravenously in divided dose to the mother. This slowed the rate to 170 beats a minute. A dose of $80 \mathrm{mg}$ verapamil three times a day with $0.25 \mathrm{mg}$ digoxin twice a day was necessary to maintain the rate at 160 beats a minute. Frusemide 40 $\mathrm{mg}$ daily was also taken by the mother. When spontaneous labour ensued at 39 weeks' gestation the fetus was in sinus rhythm and the ascites was nearly cleared. The infant required no supportive treatment but is maintained on verapamil.

Three cases of supraventricular tachycardia presented at 30,34, and 38 weeks' gestation. The onset of a "run" of supraventricular tachycardia is seen in Fig. 5. All three patients were digitalised and maintained with maternal blood levels between $1.9 \mathrm{mmol} / \mathrm{l}(1.5$ $\mathrm{mg} / \mathrm{l})$ and $2.8 \mathrm{mmol} / \mathrm{l}(2.2 \mathrm{mg} / \mathrm{l})$. Episodes of supraventricular tachycardia were not recorded after digitalisation in any case. All patients delivered normally close to term.

\section{Discussion}

Increased awareness that the fetus can be afflicted by cardiac arrhythmias has led to the referral of such cases to this centre. Twenty-three of these cases showed arrhythmias which appeared to be outside the normal patterns of fetal heart rate and rhythm variability. ${ }^{1011}$ The use of the M-mode echocardiogram has allowed the differentiation of several types of arrhythmia in these cases. 
It is possible to differentiate extrasystoles into those of atrial and those of ventricular origin. That extrasystoles occur in fetal life is well documented though their incidence varies from series to series.1213 Extrasystoles are not associated with fetal hypoxia, distress, or an adverse perinatal outcome. ${ }^{14}$ Differentiation of these from more severe arrhythmias is important and can be accomplished by the present technique.

Complete heart block is readily recognisable echocardiographically and has been diagnosed as early as 19 weeks of gestation. The necessity for accurate diagnosis of complete heart block is emphasised as this arrhythmia may be misinterpreted as fetal distress and, as happened in one case early in the series, a caesarian section may be performed unnecessarily. Furthermore, the presence of complete heart block should alert the echocardiographer to the possibility of serious structural heart disease, which was present in one third of our patients. It should also raise the question of connective tissue disease, such as systemic lupus erythematosis, in the mother.

Supraventricular tachycardias can be recognised and differentiated from other tachycardias. It is possible to treat atrial flutter and other supraventricular tachycardias in the fetus ${ }^{15} 16$ and this was successfully accomplished in this series using digoxin or digoxin and verapamil. Should digoxin not be efficacious other agents may be used such as beta adrenergic blocking drugs or procainamide. ${ }^{17}$ Variable success in the treatment of tachycardias in the fetus ${ }^{18} 19$ means that at present close monitoring and control of the maternal serum concentrations of drugs employed is essential. Failure of treatment may be the result not of inadequate maternal drug dosage but of resistance of the fetal heart to antiarrhythmic agents. It appears probable that in the presence of severe fetal cardiac failure, associated with non-immune hydrops fetalis, antiarrhythmic treatment may be less effective than in those cases where heart failure has not supervened. Fetal heart failure affects the right heart predominantly, ${ }^{20}$ causing right ventricular dilatation which can be detected echocardiographically. Thus, monitoring right ventricular size and comparison with normal standards 9 should allow treatment to be instituted before hydrops fetalis has occurred. Early recognition that an arrhythmia is present, early accurate diagnosis of the arrhythmia, and prompt well controlled drug treatment offer good prospects for continuing pregnancy to term and avoiding intrauterine and neonatal deaths from supraventricular tachycardias. Continuing the pregnancy to term rather than early delivery avoids the complications of prematurity which occurred in one of our early cases in this series. Though we have not as yet seen a ventricular tachycardia prenatally, this has been documented electrocardiographi- cally by other authors. ${ }^{1}$ Using the present M-mode echocardiographic technique it should be possible to distinguish supraventricular from ventricular tachycardias in the same way as distinguishing supraventricular from ventricular premature contractions. Accurate differentiation would avoid the inappropriate use of digoxin in a tachycardia of ventricular origin.

Accurate diagnosis of the presence and type of an arrhythmia is possible in the fetus. Echocardiographic examination allows this and also allows associated cardiac malformations to be diagnosed or excluded. Recognition of the arrhythmia and early treatment are essential if unnecessary operative or premature deliveries are to be avoided.

\section{References}

1 Shenker L. Fetal cardiac arrhythmias. Obstet Gynecol Surv 1979; 34: 561-72.

2 Allan LD, Tynan MJ, Campbell S, Wilkinson JL, Anderson RH. Echocardiographic and anatomical correlates in the fetus. Br Heart $\mathcal{F} 1980$; 44: 444-51.

3 Kleinman CS, Hobbins JC, Jaffe CC, Lynch DC, Talner NS. Echocardiographic studies of the human fetus: prenatal diagnosis of congenital heart disease and cardiac dysrhythmias. Pediatrics 1980; 65: 1059-67.

4 Allan LD, Tynan M, Campbell S, Anderson RH. Normal fetal cardiac anatomy - a basis for the echocardiographic detection of abnormalities. Prenatal Diagnosis 1981; 1: 131-9.

5 Silber DL, Durnin RE. Intrauterine atrial tachycardia: associated with massive edema in a newborn. Am $\mathcal{F}$ Dis Child 1969; 117: 722-6.

6 Kleinman CS, Donnerstein RL, Devore GR, et al. Fetal echocardiography for the evaluation of in utero congestive heart failure. $N$ Engl $\mathcal{F}$ Med 1982; 306: 568-75.

7 Kerenyi TD, Gleicher N, Meller J, et al. Transplacental cardioversion of intrauterine supraventricular tachycardia with digitalis. Lancet 1980; ii: 393-5.

8 Allan LD, Joseph MC, Boyd EGCA, Campbell S, Tynan M. M-mode echocardiography in the developing human fetus. Br Heart $\mathcal{F}$ 1982; 47: 573-83.

9 Larks SD. Fetal electrocardiography. Springfield, Illinois: Charles C Thomas, 1961:49.

10 Wheeler T, Murrills A. Patterns of fetal heart rate during normal pregnancy. Br f Obstet Gynaecol 1978; 85: 18-27.

11 Church SC, Morgan BC, Oliver TK Jr, Guntheroth WG. Cardiac arrhythmias in premature infants: an indication of autonomic immaturity? F Pediatr 1967; 71: 542-6.

12 Komáromy B, Gaal J, Lampe L. Fetal arrhythmia during pregnancy and labour. $\mathrm{Br} \mathcal{F}$ Obstet Gynaecol 1977; 84: 492-6.

13 Southall DP, Richards J, Hardwick RA, et al. Prospective study of fetal heart rate and rhythm patterns. Arch Dis Child 1980; 55: 506-11.

14 Hon EH, Huang HS. The electronic evaluation of fetal heart rate. VII. Premature and missed beats. Obstet Gynecol 1962; 20: 81-3. 
15 Harrigan JT, Kangos JJ, Sikka A, et al. Successful treatment of fetal congestive heart failure secondary to tachycardia. N Engl $\mathcal{F}$ Med 1981; 304: 1527-9.

16 Newburger JW, Keane JF. Intrauterine supraventricular tachycardia. $\mathcal{F}$ Pediatr 1979; 95: 780-6.

17 Dumesic DA. Silverman NH, Tobias S, Golbus MS. Transplacental cardioversion of fetal supraventricular tachycardia with procainamide. N Engl F Med 1982; 307: 1128-31.

18 Klein AM, Holzman IR, Austin EM. Fetal tachycardia prior to the development of hydrops - attempted pharmacological cardioversion: case report. Am $\mathcal{F}$ Obstet
Gynecol 1979; 134: 347-8.

19 Lingman G, Ohrlander S, Ohlin P. Intrauterine digoxin treatment of fetal paroxysmal tachycardia: case report. Br f Obstet Gymaecol 1980; 87: 340-2.

20 Sahn DJ, Lange LW, Allen HD, et al. Quantitative realtime cross-sectional echocardiography in the developing normal human fetus and newborn. Circulation 1980; 62: 588-97.

Requests for reprints to Dr L D Allan, Department of Paediatrics, Guy's Hospital, London SE1 9RT. 